\title{
Variabilidade da precipitação no Sertão do São Francisco, estado de Alagoas
}

\author{
Henderson S. Wanderley ${ }^{1}$, Gilberto C. Sediyama ${ }^{1}$, Flávio B. Justino ${ }^{1}$, \\ Leonidas P. de Alencar ${ }^{1} \&$ Rafael C. Delgado ${ }^{2}$
}

\begin{abstract}
Foram estudadas, neste trabalho, a homogeneidade e a existência de tendências na distribuição temporal da precipitação para a microrregião do Sertão do São Francisco, no estado de Alagoas. Os totais médios mensais de precipitação utilizados foram divididos em três períodos climatológicos: 1931-1960,1961-1990 e 1991-2011. Para essas séries temporais foram aplicados os testes estatísticos de Pettitt-Mann-Kendal e a regressão linear com o teste de Student para o coeficiente angular da reta, respectivamente, para identificar a homogeneidade e a tendência nas séries de precipitação. A distribuição temporal da precipitação para os períodos de 1931-1960 e 1961-1990 não apresentou mudanças nem tendência, as quais foram detectadas para o período de 1991-2011, com tendência significativa de aumento da precipitação em sete meses.
\end{abstract}

RESUMO

Palavras-chave: homogeneidade, tendência, mudança abrupta, mudanças climáticas

\section{Precipitation variability in the 'Sertão' of San Francisco in the State of Alagoas}

\begin{abstract}
The objective of this paper was to study the homogeneity and the existence of $t$ trends in temporal distribution of rainfall for microregion of San Francisco in the State of Alagoas. The mean precipitation data used was divided in three climatological periods: 1931-1960, 1961-1990 and 1991-2011. For these time series the statistical tests of Pettitt-Mann-Kendal was applied and linear regression with the Student test for the slope of a line, respectively to identify the homogeneity and trend in the precipitation data. The temporal distribution of precipitation for the periods 1931-1960 and 1961-1990 did not show changes or trends, which were detected for the period of 1991-2011, with a significant trend of increased precipitation in seven months.
\end{abstract}

Key words: homogeneity, trend, abrupt change, climate change 


\section{INTRODUÇÃO}

O conhecimento da variabilidade espacial e temporal da precipitação é fundamental, sobretudo para regiões que apresentam elevado índice de seca, como o Nordeste Brasileiro, que apresenta grande vulnerabilidade às alterações climáticas (Santos et al., 2010). As informações sobre mudanças no regime pluvial como resultado das mudanças climáticas são essenciais para o desenvolvimento de gestões públicas que venham conduzir ações sobre gerenciamento dos recursos hídricos e possibilitem mitigar seus impactos sobre a agricultura e o meio ambiente.

Atualmente, há uma necessidade crescente de entender como as alterações no clima estão modificando os regimes pluviométricos de uma região. O ponto de partida para este entendimento seria observar a homogeneidade de séries temporais e a existência de tendências pois uma série é considerada homogênea quando os dados pertencem à mesma população, não havendo alteração nos parâmetros (Back, 2001).

A literatura apresenta vários testes estatísticos que são utilizados para detectar a homogeneidade de séries temporais, através dos quais se verificam mudanças abruptas (quebra) na média ou na variância da distribuição da variável de interesse (Pezzatti et al., 2013). Dentre esses testes, o de Pettitt vemse destacando sobre os demais por ser menos sensível à distribuição heterogênea e a dados espúrios, sobremaneira em estudos de vazão, precipitação e temperatura (Tomozeiu et al., 2002; Biggs \& Atkinson, 2011; Villarini et al., 2011).

Embora mudanças abruptas existentes em séries temporais possam ser devidas à ação antrópica, como mudanças no local da estação e troca do instrumento de medição, dentre outros. Estudos como o de Wu (2008), Pal \& Al-Tabbaa (2009), Portela et al. (2011) e Villarini (2011) sugerem, contudo, que algumas dessas quebras, observadas em séries temporais, podem ser decorrentes da modificação do clima.

Segundo Some'e (2012) a presença de tendências em séries temporais também pode evidenciar indícios de como os dados observados estão refletindo o efeito das mudanças climáticas, sejam elas naturais ou não. A tendência pode ser entendida como uma alteração contínua e sistemática observada em uma série temporal, a qual reflete o grau de acréscimo ou diminuição nos valores da variável.

Como exemplo o estudo de Yue \& Hashino (2003) mostra tendência para a diminuição mensal da precipitação de setembro a janeiro sendo esta tendência significativa de setembro a fevereiro e de junho e julho, no Japão. Tendências significativas também foram observadas por Partal \& Kahya (2006) para os meses de janeiro, fevereiro e setembro, na Turquia.

A análise sazonal da precipitação mostrou tendências positivas e negativas no estudo de Some'e (2012) mas as tendências negativas observadas para a primavera e inverno foram maiores que aquelas apresentadas nas outras séries sazonais de modo que a tendência observada no inverno apresentou significância estatística para diminuição da precipitação no Iran, a $99 \%$ de probabilidade enquanto as demais, a 95\%, resultado análogo ao encontrado por Jain et al. (2012) para tendência sazonal da precipitação na Índia.
No Brasil, poucos estudos se dispõem a verificar a homogeneidade de séries temporais e a existência de tendência para a precipitação. Nos resultados apresentados por Minuzzi (2010) para dados mensais de precipitação em Santa Catarina, as séries pluviométricas não apresentaram descontinuidade estatisticamente significativa, nem tendência.

As séries de precipitação pluvial total anual não foram consideradas homogêneas nos resultados apresentados por Back (2001) porém apresentaram tendência significativa para o aumento da precipitação total anual para Santa Catarina. Blain (2009) não encontrou tendências significativas de ordem climática nos regimes mensais de precipitação, no estado de São Paulo.

Para a região Nordeste do Brasil ainda são escassos estudos que busquem inferir a existência de homogeneidade e de tendência em dados pluviométricos. Modelado no exposto objetivou-se, neste estudo, verificar a homogeneidade e a existência de tendências na distribuição temporal da precipitação para a microrregião do Sertão do São Francisco, estado de Alagoas.

\section{Material e Métodos}

Para o estudo foram utilizados dados mensais de precipitação compreendidos entre os anos de 1931 a 2011, provenientes do banco de dados da Agência Nacional de Águas (ANA), disponíveis para o município de Piranhas $\left(9^{\circ}\right.$ $37^{\prime} 33^{\prime \prime} \mathrm{S} ; 37^{\circ} 45^{\prime} 21^{\prime}$ 'W; $110 \mathrm{~m}$ ) localizado na microrregião do Sertão do São Francisco, estado de Alagoas.

A série de precipitação foi dividida em três períodos, conforme estabelecido pela Organização Mundial de Meteorologia (OMM) para estudos climatológicos, a saber: 1931 a 1960, 1961 a 1990 e, ainda incompleto, de 1991 a 2011.

Os períodos climatológicos foram submetidos ao teste de homogeneidade de Pettitt verificando-se se duas amostras $\mathrm{Y}_{1}, \ldots . \mathrm{Y}_{\mathrm{x}}$ e $\mathrm{Y}_{\mathrm{x}+1}, \ldots . ., \mathrm{Yx}$ pertencem à mesma população. $\mathrm{O}$ teste não-paramétrico de Pettitt é um teste estatístico utilizado para identificar o ponto de descontinuidade em uma série temporal (Yu et al., 2006). O teste tem, como hipótese nula $\left(\mathrm{H}_{\mathrm{o}}\right.$ ), a não existência de mudança brusca na série enquanto a hipótese alternativa $\left(\mathrm{H}_{1}\right)$ ela existe.

Nesse teste a série foi ordenada de $1 \mathrm{a}$ n e classificada em ordens $\left(\mathrm{O}_{i}\right)$ de acordo com sua posição de observação $\mathrm{X}_{i}$. Para cada ponto da série $\mathrm{k}$ calculou-se a soma das classes, em que $\mathrm{o}$ valor máximo de $\mathrm{k}$ determina o ponto de descontinuidade (Eqs. 1 e 2). A hipótese nula ( $\left.\mathrm{H}_{o}\right)$ será rejeitada se o valor de $\mathrm{K}_{a}$ calculado exceder a um valor crítico tabelado, em determinado nível de significância $\alpha$ (Eq.3). Para este teste foi adotado nível de significância de 0,05 .

$$
\begin{gathered}
\mathrm{U}_{\mathrm{k}}=2 \sum_{\mathrm{i}=1}^{\mathrm{k}} \mathrm{Oi}-\mathrm{k}(\mathrm{n}+1) \quad \mathrm{k}=1,2, \ldots, \mathrm{n}-1 \\
\mathrm{~K}(\mathrm{t})=\operatorname{Max}_{1 \leq \mathrm{k} \leq \mathrm{n}}\left|\mathrm{U}_{\mathrm{k}}\right| \\
\mathrm{K}_{\mathrm{a}}=\sqrt{\frac{-\ln (\mathrm{a})\left(\mathrm{n}^{3}+\mathrm{n}^{2}\right)}{6}}
\end{gathered}
$$


em que:

Uk - estatística de Pettitt

Ka - probabilidade da significância associada á rejeição de $\mathrm{H}_{\mathrm{o}}$

n - número de dados

$\mathrm{O}_{\mathrm{i}}$ - ordem da i-ésima observação, quando os valores de $\mathrm{X}_{1}, \mathrm{X}_{2} \ldots, \mathrm{X}_{\mathrm{n}}$ são ordenados em ordem crescente

As séries de precipitação foram, em seguida, submetidas ao teste de Mann-Kendall e regressão linear para verificar a existência de possíveis tendências, o qual vem apresentando resultados satisfatórios em estudo que envolve a precipitação. O teste de Mann-Kendall (Mann, 1945; Kendall, 1975) é uma análise estatística não-paramétrica que considera a hipótese de estabilidade de uma série temporal cuja sucessão de valores ocorre de forma independente e a distribuição de probabilidade deve permanecer sempre a mesma (série aleatória simples). Considerando uma série temporal $\mathrm{Y}_{\mathrm{i}}$ de $\mathrm{n}$ termos $(1 \leq \mathrm{i} \leq \mathrm{n}) \mathrm{a}$ estatística desse teste é dada pela Eq. 4.

$$
S=\sum_{i=1}^{n-1} \sum_{j=i+1}^{n} \operatorname{sinal}\left(Y_{j}-Y_{i}\right)
$$

em que:

$$
\operatorname{sinal}\left(Y_{j}-Y_{i}\right)=\left\{\begin{array}{ccc}
+1 & \text { se } & \left(Y_{j}-Y_{i}\right)>0 \\
0 & \text { se } & \left(Y_{j}-Y_{i}\right)=0 \\
-1 & \text { se } & \left(Y_{j}-Y_{i}\right)<0
\end{array}\right.
$$

Para séries com grande número de termos (n), sob a hipótese nula $\left(\mathrm{H}_{\mathrm{o}}\right)$ e ausência de tendência na população, $\mathrm{S}$ apresenta uma distribuição normal com média zero e variância definida pela Eq. 5. Testando a significância estatística de S para a hipótese alternativa $\left(\mathrm{H}_{1}\right)$ e se usando um teste bilateral $\mathrm{a}_{1}$, esta pode ser rejeitada para grandes valores da estatística z, Eq.6.

$$
\begin{aligned}
& \operatorname{Var}(S)=\frac{n(n-1)(2 n+5)}{18} \\
& Z=\left\{\begin{array}{ccc}
\frac{(S-1)}{\sqrt{\operatorname{Var}(S)}} & \text { se } & S>0 \\
0 & \text { se } & S=0 \\
\frac{(S+1)}{\sqrt{\operatorname{Var}(S)}} & \text { se } & S<0
\end{array}\right.
\end{aligned}
$$

Com base na análise da estatística $\mathrm{z}$ é feita a decisão final de aceitar ou rejeitar $\mathrm{H}_{\mathrm{o}}$, ou seja, pode-se confirmar a hipótese de estabilidade dos dados ou rejeitá-la a favor da hipótese alternativa, isto é, a de existência de tendência nos dados. $\mathrm{O}$ sinal da estatística $\mathrm{z}$ indica se a tendência é crescente $(\mathrm{z}>0)$ ou decrescente $(z<0)$. Em um teste bilateral para tendência $H_{1}$ deve ser aceita se $|z| \leq Z_{\alpha / 2}$, em que o valor de $Z_{\alpha / 2}$ é obtido da tabela na distribuição normal padronizada.
A análise de regressão foi utilizada para identificar a tendência na série temporal por meio do teste de significância do coeficiente angular da reta, Eq. 7.

$$
\mathrm{Y}=\mathrm{a}+\mathrm{bX}
$$

sendo:

Y - precipitação

$\mathrm{X}$ - tempo

a e b - coeficientes da regressão calculados pelo método dos mínimos quadrados

Considerando a regressão linear de Y com variável aleatória no tempo $\mathrm{X}$, a hipótese nula $\left(\mathrm{H}_{\mathrm{o}}\right)$ de que não existe uma tendência, é testada por meio do teste $\mathrm{t}$ de Student com n-2 graus de liberdade, Eq. 8. A hipótese de que não há tendência é rejeitada quando o valor de t calculado é maior, em valor absoluto, do que o valor crítico $t_{\alpha / 2, n-2}$, tabelado, a um nível de significância $\alpha$. Para os cálculos dos testes de tendências foram adotados os níveis de significância de 10 e 5\%.

$$
t=\frac{r \sqrt{n-2}}{\sqrt{1-r^{2}}}=\frac{b}{s / \sqrt{S S x}}
$$

em que:

n - tamanho da amostra

r $\quad$ - coeficiente de correlação de Pearson

S - desvio padrão dos resíduos

b - coeficiente angular da reta

$\mathrm{SS}_{\mathrm{X}}$ - soma dos quadrados da variável independente (tempo em análise de tendência)

\section{Resultados e Discussão}

O regime de chuvas comportou-se de forma análoga entre períodos climatológicos em estudo, com pequena variação mensal da precipitação para quase todos os meses. No entanto, observou-se que nos meses de abril e maio, para o período de 1991 a 2011, houve uma redução da precipitação de e 45,39 e $22,17 \%$, respectivamente, para esses meses, em comparação com o período de 1961 a 1990 (Figura 1).

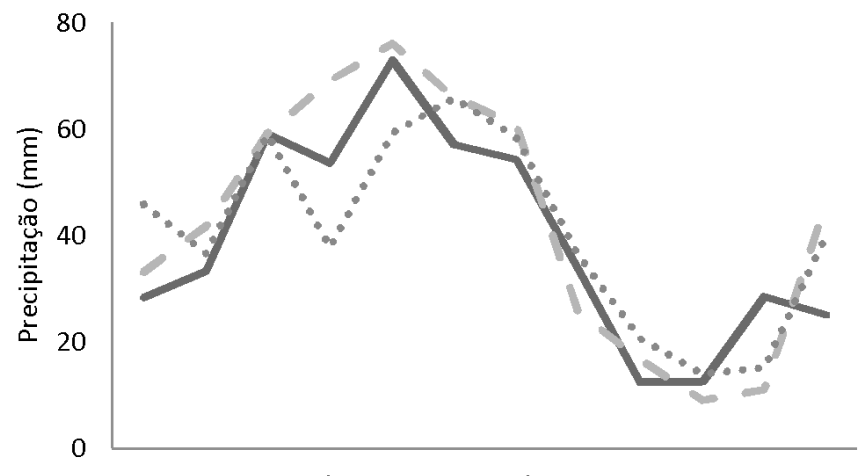

Jan Fev Mar Abr Mai Jun Jul Ago Set Out Nov Dez

1931-1960 - 1961-1990

1991-2011

Figura 1. Distribuição temporal da precipitação para os períodos climatológicos em estudo 
A redução na precipitação para esses meses, pode acarretar transtorno para a agricultora e para o armazenamento de água no solo uma vez que os meses de abril e maio estão inseridos na quadra chuvosa desta região juntamente com os meses de junho e julho (Molion \& Bernardo, 2002). No período compreendido entre os anos de 1961 a 1990, a precipitação para os meses inseridos na quadra chuvosa foi sempre superior a $60 \mathrm{~mm}$; mesmo assim, para o período 1991 a 2011 apenas no mês de junho a precipitação apresentou índice superior a $60 \mathrm{~mm}$.

A distribuição da precipitação mensal entre os anos de 1931 a 1960, mostrou-se homogênea, não sendo detectado ponto de descontinuidade na série (Tabela 1). Além da homogeneidade observada na distribuição da precipitação não foi verificada a existência de tendência estaticamente significativa na distribuição.

Tabela 1. Testes estatísticos aplicados à precipitação para o período de 1931-1960

\begin{tabular}{ccrrr}
\hline Meses & Pettitt & \multicolumn{1}{c}{$\mathbf{Z}$} & $\mathbf{b}_{1}$ & $\mathbf{t}$ \\
Jan & HM & 1,03 & 0,7132 & 0,82 \\
Fev & HM & $-1,26$ & $-0,9104$ & $-1,45$ \\
Mar & HM & 0,99 & 3,1948 & 1,94 \\
Abr & HM & 0,49 & 0,4235 & 0,44 \\
Mai & HM & $-0,85$ & $-0,6918$ & $-0,77$ \\
Jun & HM & $-0,64$ & $-0,5873$ & $-0,80$ \\
Jul & HM & $-1,25$ & $-0,6091$ & $-0,86$ \\
Ago & HM & $-1,05$ & $-0,5582$ & $-1,07$ \\
Set & HM & $-0,14$ & $-0,0344$ & $-0,10$ \\
Out & HM & $-0,16$ & $-0,2731$ & $-0,89$ \\
Nov & HM & 1,03 & 1,0308 & 1,13 \\
Dez & HM & $-0,26$ & $-0,2974$ & $-0,50$ \\
\hline
\end{tabular}

HM - homogênea; Z - Teste de Mann-Kendall; $b_{1}$ - declinação da reta, t - teste de Student

A precipitação, porém, mostrou uma redução entre os meses de maio a outubro indicada pelo teste de Mann-Kendall mas esta redução foi inferior a $10 \mathrm{~mm}_{\text {década }}{ }^{-1}$. Para este período (1931-1960) o único mês que apresentou mudança maior que $10 \mathrm{~mm}$ década $^{-1}$ foi março, com um aumento de mais de 30 $\mathrm{mm}$ por década, porém sem significância estatística.

Os resultados encontrados para o período climatológico compreendido entre os anos de 1961 a 1990 foram análogos aos encontrados para o período climatológico anterior (19311960), não sendo verificada, na série de precipitação, quebra ou tendência estaticamente significativa (Tabela 2).

Apenas os meses de fevereiro, março, julho e agosto mostraram acréscimo nos totais pluviométricos. Para o mês de março novamente se observou aumento na distribuição da precipitação com mais de $25 \mathrm{~mm}_{\text {década }}^{-1}$. Os demais meses apresentaram diminuição da precipitação sendo os meses de abril e maio os que apresentaram os maiores índices de redução, respectivamente, 13 e $15 \mathrm{~mm}_{\text {década }}{ }^{-1}$.

Esses resultados mostram que os meses de transição do período seco para chuvoso, fevereiro e março, foram mais chuvosos para este período climatológico, embora os meses do período chuvoso tenham demonstrado a maior redução pluviométrica para a série.

A redução da precipitação para este período climatológico também foi observada por Silva (2004). Dentre as 14 estações estudadas para o Nordeste do Brasil, 11 sinalizaram tendência
Tabela 2. Testes estatísticos aplicados à precipitação para o período de 1961-1990

\begin{tabular}{ccrrr}
\hline Meses & Pettitt & \multicolumn{1}{c}{$\mathbf{Z}$} & \multicolumn{1}{c}{$\mathbf{b}_{\mathbf{1}}$} & $\mathbf{t}$ \\
Jan & HM & $-0,77$ & $-0,8193$ & $-1,12$ \\
Fev & HM & 0,28 & 0,3527 & 0,37 \\
Mar & HM & 1,53 & 2,5738 & 2,02 \\
Abr & HM & $-0,71$ & $-1,3131$ & $-0,91$ \\
Mai & HM & $-1,19$ & $-1,5550$ & $-1,22$ \\
Jun & HM & $-0,53$ & $-0,2823$ & $-0,30$ \\
Jul & HM & 0,58 & 0,1760 & 0,19 \\
Ago & HM & 0,33 & 0,0925 & 0,28 \\
Set & HM & $-0,25$ & $-0,0046$ & $-0,01$ \\
Out & HM & $-0,52$ & $-0,1399$ & $-0,58$ \\
Nov & HM & $-0,73$ & $-0,3470$ & $-0,94$ \\
Dez & HM & $-0,29$ & $-1,3141$ & $-0,76$ \\
\hline
\end{tabular}

HM - homogênea; $\mathrm{Z}$ - Teste de Mann-Kendall; $b_{1}$ - declinação da reta, $\mathrm{t}$ - teste de Student

para o decréscimo da precipitação até a década de 90. Apesar do estudo não contemplar nenhuma estação localizada no estado de Alagoas, a estação localizada no Recife (estação mais próxima) demonstrou tendência estaticamente significativa a $1 \%$, com decréscimo de $28,8 \mathrm{~mm}_{\text {década }}{ }^{-1}$.

Para os anos compreendidos entre 1991 a 2011 os resultados se mostraram antagônicos aos observados nos outros períodos em estudo. Comumente, as outras séries analisadas mostraram quatro meses com aumento da precipitação, com os demais meses apresentando diminuição. No recente período foram observados oito meses com tendência de aumento na precipitação, sendo sete estatisticamente significativos (Tabela 3). Os resultados obtidos por meio do teste $t$ apresentaram significância a 5\% para os meses de maio e setembro, ratificando o aumento da precipitação encontrada pelo teste de Mann-Kendall.

Tabela 3. Testes estatísticos aplicados à precipitação para o período de 1991-2011

\begin{tabular}{clccc}
\hline Meses & Pettitt & $\mathbf{Z}$ & $\mathbf{b}_{1}$ & $\mathbf{t}$ \\
Jan & $\mathrm{HM}$ & $-1,08$ & $-0,94$ & $-0,38$ \\
Fev & $\mathrm{NH}^{\star \star}$ & $2,06^{\star \star}$ & 2,18 & 1,59 \\
Mar & $\mathrm{HM}$ & $1,69^{\star}$ & 1,66 & 0,74 \\
Abr & $\mathrm{HM}$ & $1,87^{\star}$ & 1,77 & 1,35 \\
Mai & $\mathrm{NH}^{\star \star}$ & $2,38^{\star \star}$ & 4,38 & $2,80^{\star \star}$ \\
Jun & $\mathrm{HM}$ & 0,54 & 0,15 & 0,11 \\
Jul & $\mathrm{HM}$ & $-0,57$ & 0,44 & 0,36 \\
Ago & $\mathrm{HM}$ & $1,69^{\star}$ & 1,50 & 1,85 \\
Set & $\mathrm{HM}$ & $1,93^{\star}$ & 1,57 & $2,77^{\star \star}$ \\
Out & $\mathrm{HM}$ & $1,37^{\star}$ & 0,32 & 0,47 \\
Nov & $\mathrm{HM}$ & $-1,41$ & $-1,03$ & $-1,25$ \\
Dez & $\mathrm{HM}$ & $-0,21$ & 0,19 & 0,09 \\
\hline
\end{tabular}

HM - homogênea; NH - não homogênea; Z - Teste de Mann-Kendall; $b_{1}$ - declinação da reta; $\mathrm{t}$ - teste de Student; ${ }^{*}$ significativo a $0,10,{ }^{*}$ significativo a 0,05

Um aumento na precipitação de cerca de $2 \%$, por década, também foi observado para o hemisfério sul, entre os paralelos $0-55^{\circ} \mathrm{S}$. Para grande parte do hemisfério norte este aumento foi de $0,5-1 \%$ por década mas para as altas latitudes observouse um aumento médio anual entre 7-12\% (Xu et al., 2003).

Além dos aumentos significativos para a precipitação no período 1991-2011, os meses de fevereiro e maio apresentaram pontos de descontinuidade estatisticamente significativos.

O mês de fevereiro mostrou descontinuidade na série de precipitação no ano de 2002. Entre os anos de 1991 a 2001 a precipitação média mensal para fevereiro foi de 20,17 
mm passando para $54,83 \mathrm{~mm}$ deste ano até o final da série caracterizando um aumento na média pluviométrica mensal de $271,8 \%$ (Figura 2).

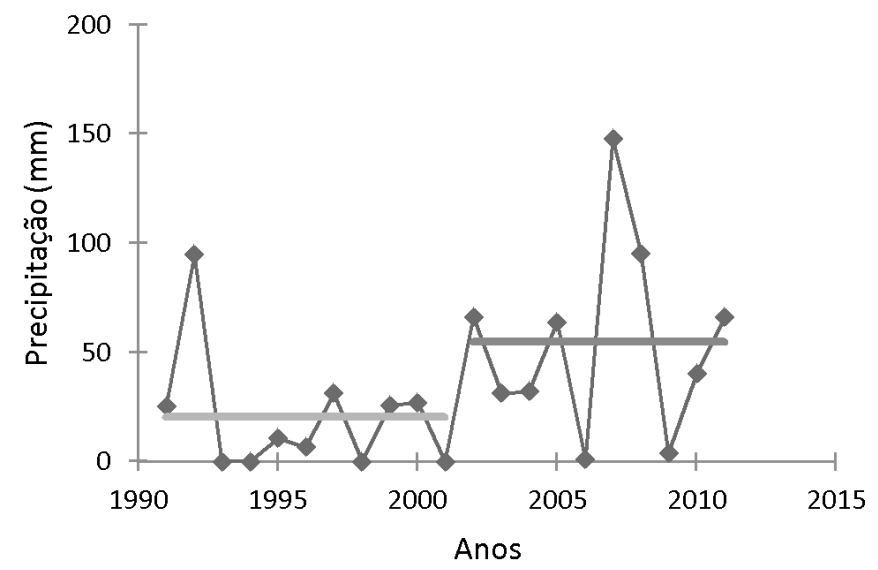

Figura 2. Distribuição temporal e o ponto de descontinuidade da série de precipitação para o mês de fevereiro

O acréscimo na precipitação não contemplou todos os meses de fevereiro após o ponto de descontinuidade devido à existência de alguns episódios de secas, como as observadas em 2006 e 2009 na região em estudo. Algumas dessas secas provocam grandes impactos para a região semiárida com a de 2012, uma das maiores dos últimos 50 anos.

No mês de maio o ponto de descontinuidade foi observado em 2005 (Figura 3). A precipitação, antes e após este ponto, foi de 35,62 e 106,61 mm, respectivamente, representando um aumento de $299,2 \%$.

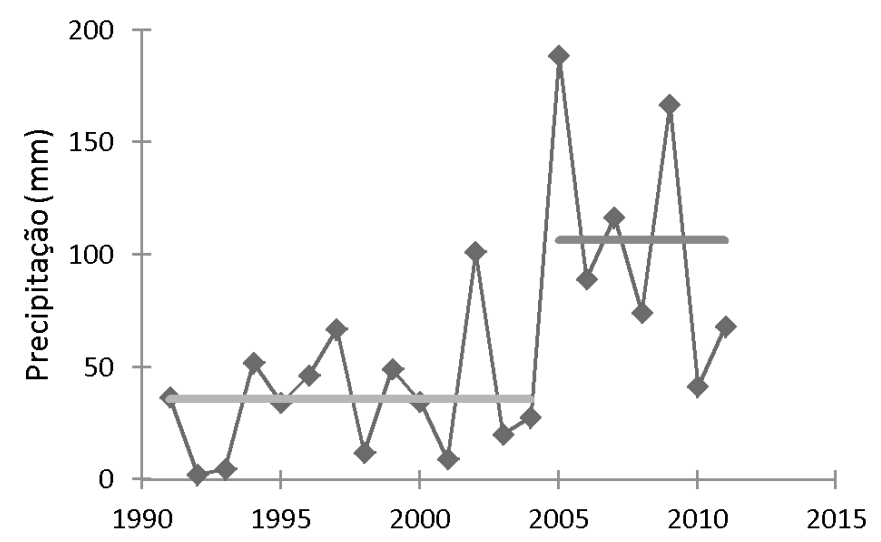

Figura 3. Distribuição temporal e o ponto de descontinuidade da série de precipitação para o mês de maio

Por não se dispor de informações sobre mudanças ocorridas na estação em estudo, não pode ser conclusiva que a mudança observada na precipitação nos meses de fevereiro e maio, tenha sido decorrente da ação antrópica, embora possa ter ocorrido. No entanto, estudos como o de Montenegro \& Ragab (2012) apontam para um incremento da precipitação para a costa leste do Nordeste de $25,25 \%$ até 2039 , em decorrência da intensificação do ciclo hidrológico em função do aumento da temperatura, quando considerados os cenários divulgados pelo IPCC em 2007.
Os resultados sugerem que o déficit na precipitação observado no período de 1991-2011 (Figura 1) sobremaneira para os meses inseridos na quadra chuvosa de Alagoas, pendurou, em média, até o fim da década de 90, início dos anos 2000; após este período se observa aumento na pluviometria, em Alagoas.

O aumento observado na precipitação, na localidade em estudo, pode estar refletindo um acréscimo das chuvas no agreste, sertão e sertão do São Francisco alagoano, pois na classificação climática do comportamento pluviométrico realizada por Souza et al. (1992) os municípios localizados nessas regiões, incluindo o de estudo, foram classificados como pertencentes à mesma região climatológica.

Este fato pode estar relacionado ao aumento da temperatura que, por sua vez, tende a acelerar o ciclo hidrológico originando mais evaporação e precipitação. Um aquecimento maior tende a alterar os padrões de circulação, tanto em escala global quanto regional, o que pode ser uma das causas das variações já observadas na distribuição espacial e temporal, encontradas na precipitação em Alagoas (Pal \& Al-Tabbaa, 2009).

A distribuição das chuvas no Nordeste sofre influência da interação dos oceanos Pacíico e Atlântico, como a Oscilação Decadal do Pacífico (ODP), Dipolo do Atlântico e El Niño/ Oscilação Sul (ENOS). Destacam-se, também, outros sistemas indutores de chuva na região, como a Zona de Convergência Intertropical (ZCIT), Sistemas Frontais, Perturbações Ondulatórias no campo dos Alísios (POA's), Linhas de Instabilidade (LI), Vórtices Ciclônicos em Altos Níveis (VCAN), Onda de Leste (OL) e os Complexos Convectivos de Meso escala (CCM).

Além desses sistemas ocorre, também, a contribuição por circulações orográficas e células convectivas. Por sofrer a influência de sistemas produtores de chuvas de macro, meso e micro escala, qualquer distúrbio que ocorra em um destes sistemas, seja em função do aumento da temperatura ou não, tem o potencial para modificar a distribuição das chuvas no Nordeste.

A resposta da modificação de um desses sistemas na distribuição da precipitação, é observada nos resultados encontrados por Brito et al. (2011) que verificaram um aumento de três vezes na frequência das trovoadas no estado de Alagoas após o ano de 1998, em decorrência do início da fase fria da Oscilação Decadal do Pacífico (ODP). Essas trovoadas estão associadas a anomalias da temperatura da superfície do mar quando negativa (positiva) e, associadas ao evento La Ninã (El Niño) houve um aumento (diminuição) de $80 \%(20 \%)$ dos dias com trovoada, em anos de neutralidade o comportamento é análogo ao de La Ninã. Nos anos de 2002 e 2005, como exemplo, foram observados 16 e 17 eventos de trovoadas em Alagoa, respectivamente, enquanto no ano de 1996 foram observados apenas seis casos.

Outro resultado que corrobora com os demais já apresentados se dá pelo acréscimo do número de casos de precipitação intensa em Alagoas; somente entre os anos de 2003 a 2006 foram registrados 21 casos (Silva et al., 2011). Além do mais, em Alagoas os anos de 2003, 2010 e 2011 foram caracterizados por apresentar grandes quantidades de chuvas, pois causaram enchentes em diversas cidades alagoanas e pernambucanas, com destruição total e/ou parcial. 
Só em maio de 2011 a precipitação foi de até $200 \mathrm{~mm}$ acima da média para algumas regiões da costa leste do Nordeste; só no estado de Pernambuco as chuvas intensas deixaram 144.000 pessoas desabrigadas (AMS, 2012).

$\mathrm{O}$ aumento pluviométrico ratificado neste estudo mostrouse irregular na escala temporal já que esta tendência não foi observada em todos os meses. $\mathrm{O}$ aumento das chuvas, em sua grande parte, é decorrente da intensificação do número de eventos extremos e enchentes, resultantes da modificação dos sistemas produtores de chuva na região em estudo que, por sua vez, pode estar refletindo distúrbios em decorrência de um aquecimento maior.

Com isto, setores como a agricultura, pecuária e turismo, poderão ser afetados pelas condições de tempo e clima.

\section{Conclusões}

1. A distribuição da precipitação para os períodos compreendidos entre os anos de 1931-1960 e 1961-1990 foi homogênea, não sendo verificada existência de tendências.

2. Para o período compreendido entre os anos 19912011 os meses de fevereiro e maio apresentaram pontos de descontinuidade da precipitação nos anos de 2002 e 2005, respectivamente.

3. A precipitação no período de 1991-2011 demostrou tendência estaticamente significativa de aumento da precipitação em sete meses.

\section{Literatura Citada}

AMS - American Meteorological Society. State of the climate in 2011. Special Supplement to the Bulletin of the American Meteorological Society, v.93, p.5-354, 2012.

Back, A. J. Aplicação de análise estatística para identificação de tendências climáticas. Pesquisa Agropecuária Brasileira, v.36, p. 717-726, 2001.

Blain, G. C. Considerações estatísticas relativas a oito séries de precipitação pluvial da secretaria de agricultura e abastecimento do estado de São Paulo. Revista Brasileira de Meteorologia, v.24, p.12-23, 2009.

Biggs, E. M.; Atkinson, P. M. A characterization of climate variability and trends in hydrological extremes in the Severn Uplands. International Journal of Climatology, v.31, p.1634-1652, 2011.

Brito, B. M.; Levit, V.; Fedorova, N.; Molion, L. C. B.; Tenório, R. S.; Rodrigues, R. N.; Silva, B. F. P. Análise do comportamento das trovoadas no estado de Alagoas, previsão à curto prazo. Revista Brasileira de Meteorologia, v.26, p.243-256, 2011.

Jain, S. K.; Kumar, V.; Saharia, M. Analysis of rainfall and temperature trends in northeast India. International Journal of Climatology, v.32, p.1-11, 2012.

Kendall, M. G. Rank correlation methods. 4.ed. London: Charles Griffin, 1975. 196p.

Mann, H. B. Non-parametric test against trend. Econometrika, v.13, p.245-259, 1945.

Minuzzi, R. B. Tendências na variabilidade climática de Santa Catarina, Brasil. Revista Brasileira de Engenharia Agrícola e Ambiental, v.14, p.1288-1293, 2010.
Molion, L.C.B.; Bernardo, S.O. Uma revisão da dinâmica das chuvas no Nordeste Brasileiro. Revista Brasileira de Meteorologia, v.17, p.1-10, 2002.

Montenegro, S.; Ragab, R. Impact of possible climate and land use changes in the semi arid regions: A case study from North Eastern Brazil. Journal of Hydrology, v.434, p.55-68, 2012.

Pal, I; Al-Tabbaa, A. Trends in seasonal precipitation extremes - An indicator of 'climate change' in Kerala, India. Journal of Hydrology, v.367, p.62-69, 2009.

Partal, T.; Kahya, E. Trend analysis in Turkish precipitation data. Hydrological Processes, v.20, p.2011-2026, 2006.

Pezzatti, G.B.; Zumbrunnen, T.; Bürgi, M.; Ambrosetti, P.; Conedera, M. Fire regime shifts as a consequence of fire policy and socio-economic development: An analysis based on the change point approach. Forest Policy and Economics, v.29, p.7-18,2013.

Portela, M. M.; Quitela, A, C.; Santos, J. F.; Vaz, C.; Martins, C. Tendência em séries temporais de variáveis hidrológicas. Revista Recursos Hídricos, v.32, p.43-60, 2011.

Santos, D. N; Silva, V. P. R.; Sousa, F. A. S.; Silva, R. A. Estudo de alguns cenários climáticos para o Nordeste do Brasil. Revista Brasileira de Engenharia Agrícola e Ambiental, v.14, p.492-500, 2010.

Silva, B. F. P.; Fedorova, N.; Levit, V.; Peresetsky, A.; Brito, B. M. Sistemas sinóticos associados às precipitações intensas no estado de Alagoas. Revista Brasileira de Meteorologia, v.26, p.323-338, 2011.

Silva, V. P. R. On climate variability in Northeast of Brazil. Journal of Arid Environments, v.58, p.575-596,2004.

Some'e, B. S.; Ezani, A.; Tabari, H. Spatiotemporal trends and change point of precipitation in Iran. Atmospheric Research, v.133, p.1-12, 2012.

Souza, J. L.; Amorim, R. F. C.; Carvalho, S. M. R.; Pereira, J. O.; Curi, P. R. Agrupamento de estações pluviométricas do estado de Alagoas, utilizando-se análise multivariada. Revista Brasileira de Meteorologia, v.7, p.603-612, 1992.

Tomozeiu, R.; Busuioc, A.; Stefan, S. Changes in seasonal mean maximum air temperature in Romania and their connection with large-scale circulation. International Journal of Climatology, v.22, p.1181-1196, 2002.

Villarini, G. Analyses of annual and seasonal maximum daily rainfall accumulations for Ukraine, Moldova, and Romania. International Journal of Climatology, v.32, p.2213-2226, 2011.

Villarini, G.; Smith, J.A.; Baeck, M.L.; Krajewski, W.F. Examining flood frequency distributions in the Midwest U.S. Journal of the American Water Resources Association, v.43, p.447-463, 2011.

$\mathrm{Wu}, \mathrm{Y}$. Simultaneous change point analysis and variable selection in a regression problem. Journal of Multivariate Analysis, v.99, p.2154-2171, 2008.

Xu, Z. X.; Takeuchia, K.; Ishidaira, H. Monotonic trend and step changes in Japanese precipitation. Journal of Hydrology, v.279, p.144-150, 2003.

Yu, P. S.; Yang, T. C.; Kuo, C. C. Evaluating long-term trends in annual and seasonal precipitation in Taiwan. Water Resources Management, v.20, p.1007-1023, 2006.

Yue, S.; Hashino, M. Long term trends of annual and monthly precipitation in Japan. Journal of the American Water Resources Association, v.39, p.587-596, 2003. 